\title{
Fatores de risco relacionados a má evolução no tratamento da infecção periprótese não convencional
}

\section{Risk Factors Related to Poor Outcomes of the Treatment of Non-conventional Periprosthetic}

\author{
Dan Carai Maia Viola ${ }^{1,2}$ Henrique Ribeiro Rodrigues Neto ${ }^{1(} \quad$ Jairo Greco Garcia ${ }^{10}$ \\ Marcelo de Toledo Petrilli ${ }^{10}$ Fabianne Altruda de Moraes Costa Carlesse ${ }^{3,4}$ \\ Reynaldo Jesus-Garcia Filho ${ }^{1,2}$
}

${ }^{1}$ Instituto de Oncologia Pediátrica (IOP/GRAACC), Departamento de Ortopedia e Traumatologia,Universidade Federal de São Paulo (UNIFESP), São Paulo, SP, Brasil

2 Programa de Ortopedia, Hospital Israelita Albert Einstein, São Paulo, SP, Brasil

${ }^{3}$ Departamento de Pediatria, Universidade Federal de São Paulo (UNIFESP), São Paulo, SP, Brasil

${ }^{4}$ Instituto de Oncologia Pediátrica (IOP), Grupo de Apoio ao Adolescente e à Criança com Câncer (GRAACC), São Paulo, SP, Brasil
Endereço para correspondência Dan Carai Maia Viola, MD, PhD, Departamento de Ortopedia e Traumatologia,Universidade Federal de São Paulo, Rua Botucatu, 740, Vila Clementino, São Paulo, SP, 04023-062, Brasil (e-mail: dcmviola@gmail.com).

Rev Bras Ortop 2021;56(5):615-620.

\section{Resumo \\ Palavras-chave \\ - próteses e implantes \\ - sarcoma \\ - infecções \\ - osteossarcoma \\ - amputação \\ - desbridamento \\ Objetivos O objetivo do estudo é identificar os principais fatores de risco relaciona- dos a má evolução do tratamento da infecção periprótese. \\ Métodos Foram avaliados de forma retrospectiva os prontuários de 109 pacientes submetidos a cirurgias de endoprótese não convencional (primárias e revisões), no período de 01 de janeiro de 2007 a 31 de dezembro de 2018. Destes, 15 pacientes diagnosticadas com infecção periprótese foram elegíveis para a participação no estudo. As variáveis sexo, idade do diagnóstico, osso acometido, duração da cirurgia, contagem de leucócitos no pré-operatório, infecção do trato urinário no $1^{\circ}$ ano pós- operatório e tempo decorrido entre a colocação da endoprótese e o diagnóstico da infecção foram relacionadas aos desfechos utilizando o Teste Exato de Fisher (variáveis bicategóricas) e o Teste Anova (variáveis tricategóricas). A média de tempo entre diagnóstico e desfecho foram comparadas pelo método de t-student.}

Trabalho desenvolvido no Instituto de Oncologia Pediátrica (IOP/GRAACC)/ Departamento de Ortopedia e Traumatologia (DOT) da Universidade Federal de São Paulo (UNIFESP), São Paulo, SP, Brasil. recebido

12 de Junho de 2020

aceito

08 de Janeiro de 2021
DOI https://doi.org/

$10.1055 / \mathrm{s}-0041-1731354$. ISSN 0102-3616. (c) 2021. Sociedade Brasileira de Ortopedia e Traumatologia. All rights reserved.

This is an open access article published by Thieme under the terms of the Creative Commons Attribution-NonDerivative-NonCommercial-License, permitting copying and reproduction so long as the original work is given appropriate credit. Contents may not be used for commercial purposes, or adapted, remixed, transformed or built upon. (https://creativecommons.org/ licenses/by-nc-nd/4.0/)

Thieme Revinter Publicações Ltda., Rua do Matoso 170, Rio de Janeiro, RJ, CEP 20270-135, Brazil 


\begin{abstract}
Keywords

- prosthesis and implants

- sarcoma

- infections

- osteosarcoma

- amputation

- debridement

Objectives This study aimed to identify the main risk factors related to poor outcomes after periprosthetic infection treatment.

Methods Medical records from 109 patients who underwent non-conventional endoprosthesis surgeries (primary and revision procedures) from January 1, 2007 to December 31, 2018, were retrospectively evaluated. Fifteen patients diagnosed with periprosthetic infection were eligible for study participation. Variables, including gender, age at diagnosis, affected bone, surgery duration, white blood cell (WBC) count before endoprosthesis placement, urinary tract infection during the $1^{\text {st }}$ postoperative year, and time elapsed from endoprosthesis placement to infection diagnosis, were related to outcomes using the Fisher's exact test (for bicategorical variables) or ANOVA (for tricategorical variables). Mean times from diagnosis to final outcome were compared using the Student's t-test.

Results These risk factors did not show a statistically significant correlation with outcomes. Data revealed a trend for difference between the mean time for infection onset and the final outcome. Due to the limited sample, we believe that studies with larger cohorts can prove this trend.

Conclusion We identified that the time from endoprosthesis placement to infection symptoms onset tends to be related to the outcome and patient evolution during periprosthetic infection treatment. Although apparently correlated, other associated factors were not statistically linked to poor treatment outcomes.
\end{abstract}

Resultados Os fatores de risco avaliados não demostraram correlação estatisticamente significante com os desfechos. Os dados demonstram haver tendência de diferença entre a média de tempo do aparecimento do processo infeccioso e o desfecho final do paciente. Devido a amostra limitada, acreditamos que estudos com coortes maiores possam comprovar essa tendência.

Conclusão Identificamos que o tempo entre a cirurgia de colocação da endoprótese e o aparecimento dos sintomas de infecção tende a ter relação com o desfecho e a evolução do paciente no tratamento da infecção periprótese. Os demais fatores associados, apesar de aparentemente relacionados, também não se mostraram estatisticamente relacionados a má evolução no tratamento.

\section{Introdução}

A sobrevida dos pacientes com tumores ósseos malignos tem aumentado a partir dos avanços em quimioterapia e radioterapia. Apesar de preferencialmente ser indicado reconstruções biológicas, em muitas situações, faz-se necessário a utilização de técnicas não biológicas com a utilização de endopróteses não convencionais. Como qualquer artroplastia convencional, as endopróteses apresentam susceptibilidade a falhas e infecção. ${ }^{1,2}$

As falhas das próteses ocorrem por soltura asséptica, falha de partes moles, falhas estruturais, progressão tumoral e infecção. ${ }^{3} \mathrm{~A}$ infecção da endoprótese é uma das principais complicações deste tipo de cirurgia, com incidência reportada de 2 a $19,5 \%{ }^{4}$

O risco de infecção é diferente conforme a localização do tumor primário. As ressecções ao redor do joelho (tíbia proximal e fêmur distal), apresentam um risco aumentado para infecção em comparação com úmero. ${ }^{5}$

A presunção do diagnóstico da infecção periprótese ocorre através do quadro clínico. $O$ exame de ultrassom pode detectar coleção ao redor da prótese e os exames de radiografia podem ou não evidenciar sinais de soltura do implante. As principais alterações laboratoriais são a modificação do leucograma (com padrão infeccioso), aumento da velocidade de hemossedimentação e elevação da proteína $C$ reativa sérica. ${ }^{6}$

Vários estudos preconizam como critério diagnóstico a presença de ao menos um dos seguintes: (1) crescimento do mesmo microrganismo em duas ou mais amostras do líquido sinovial ou dos tecidos peri-implante colhidos em cultura, local do implante ou líquido sinovial com secreção purulenta; (2) sinais de inflamação aguda no exame histopatológico dos tecidos peri-implante; ou (3) presença de fístula comunicando com a endoprótese. ${ }^{4}$

O patógeno mais frequentemente identificado nas culturas é o estafilococos coagulase-negativo (30 a 43\% dos casos) e o estafilococos aureus (12 a 23\%), seguidos por flora mista (10-11\%), estreptococos (9-10\%), bacilos gram-negativos (3$6 \%)$, enterococos (3-7\%) e anaeróbios $(2-4 \%) .{ }^{7-10}$ Existe uma relação entre topografia da endoprótese e patógeno. $O P$. acnes, por exemplo, é o principal causador de infecção no 
pós-operatório de endoprótese de ombro, o que justifica um maior estudo para se isolar o microrganismo causador afim de aumentar as chances de cura., ${ }^{71}$

O biofilme é o principal fator que dificulta o tratamento da infecção nos implantes. Este é definido como um conjunto de bactérias que são encapsuladas dentro de sua própria matriz polimérica, que atinge uma massa crítica sobre o implante contaminado, induz uma reação inflamatória em seu hospedeiro, e que pode levar, em uma última análise, a falha do implante. A bactéria dentro do biofilme é significantemente menos suscetível à antibiótico, a defesa do hospedeiro e antissépticos, característica essa que a faz mais difícil de tratar. Uma vez que o biofilme leve a falha do implante, as opções clínicas são bastante limitadas e envolve a supressão com antibiótico por um longo período ou a revisão cirúrgica, o que leva a grandes morbidades e em alguns casos ao óbito. ${ }^{8}$

A incidência de infecção periprótese é maior nos pacientes submetidos a ressecção tumoral do que nos pacientes submetidos a artroplastias por outras causas que não oncológicas. $^{9,11,12}$ Isso se deve a inúmeras diferenças, como os implantes apresentarem maior área de superfície, maior via de acesso, frequentemente duração da cirurgia maior, maior perda de sangue, espaço morto, imunodeficiência em decorrência de quimioterapia, radioterapia, condições ruins de partes moles e ressecção da articulação de modo extraarticular. $^{10,13,14}$

A localização da neoplasia também é importante, sendo a tíbia proximal e a endoprótese pélvica, fatores de risco para infecções endoprotéticas. As cirurgias pélvicas, pela sua dificuldade técnica, apresentam tempo cirúrgico prolongado, enquanto a tíbia proximal é suscetível à infecção devido a dificuldade em conseguir boa cobertura de partes moles. ${ }^{9}$

Encontramos na literatura a correlação da infecção periimplante com outros fatores, como: idade, dermatite atópica, diabetes, obesidade artrite reumatoide, tabagismo, histórico de infecção, sexo masculino, deiscência e hematoma. ${ }^{3,13}$ Entretanto, não há uma definição sobre quais fatores realmente estão associados à evolução do paciente após o tratamento da infecção.

O objetivo do estudo é identificar os principais fatores de risco relacionados a má evolução do tratamento da infecção periprótese.

\section{Material e Método}

Foram avaliados de forma retrospectiva os prontuários de 109 pacientes submetidos a cirurgias de endoprótese não convencional (primárias e revisões), no período de 01 de janeiro de 2007 a 31 de dezembro de 2018. Destes, 16 foram diagnosticados com infecção periprótese. Dos 16 pacientes avaliados, uma havia sido submetida a cirurgia inicial e diagnóstico de infecção em outro serviço e foi retirada da análise estatística, restando um grupo de 15 pacientes $(13,7 \%)$ com infecção periprótese.

O trabalho consistiu na análise retrospectiva de prontuários dos pacientes com diagnóstico de infecção periprótese submetidos ao tratamento na Instituição. O trabalho foi aprovado pelo Comitê de Ética em Pesquisa e está registrado na Plataforma Brasil sob o número CAAE 12665419.2.0000.5505.

\section{Diagnóstico de Infecção}

Consideramos para o diagnóstico de infecção periprótese a combinação de sinais e sintomas classicamente estabelecidos sugestivos de processo infeccioso ativo: dor ao redor da prótese e/ou no membro sem outra causa diagnosticável, aumento de temperatura em relação ao membro contralateral, hiperemia local, edema e febre.

Após a suspeita clínica, todos pacientes foram submetidos a exame de ultrassonografia com comprovação de coleção líquida ao redor da endoprótese. Utilizamos, por rotina, coleta de exames laboratoriais séricos de hemocultura, leucograma, velocidade de hemossedimentação e dosagem de proteína $\mathrm{C}$ reativa para auxiliar no diagnóstico e avaliação do tratamento do processo infeccioso.

\section{Tratamento do processo infeccioso}

A partir do diagnóstico clínico de infecção, os pacientes foram submetidos ao tratamento preconizado pelo protocolo do nosso serviço, com limpeza cirúrgica e desbridamento precoce de tecidos desvitalizados - em até 7 dias após o diagnóstico clínico do processo infeccioso periprótese. Durante a cirurgia de limpeza cirúrgica foram colhidas amostras do líquido periprótese para cultura.

Após a cirurgia de limpeza cirúrgica, iniciamos o tratamento com antibioticoterapia endovenosa. Usualmente utilizamos cefalosporina de $1^{1}$ geração até o resultado das culturas colhidas. Após o resultado das culturas, o tratamento é direcionado conforme o perfil de antibioticoterapia para o agente etiológico isolado. Os pacientes que, em até 15 dias, não evoluíram com melhora dos sinais, sintomas e exames laboratoriais apresentaram indicação de revisão da endoprótese.

Utilizamos, preferencialmente, a revisão em dois tempos com a utilização de espaçador de cimento acrílico com antibiótico por 45 a 180 dias e subsequente retirada do espaçador e revisão com nova endoprótese não convencional. ${ }^{6}$ Todos pacientes foram informados sobre a alternativa do procedimento de amputação do membro.

Um paciente foi submetido a revisão em único tempo, com retirada dos componentes da endoprótese, nova limpeza cirúrgica e desbridamento, e subsequente colocação de um novo implante no mesmo tempo cirúrgico. ${ }^{15}$

Após a cirurgia de revisão da endoprótese, os pacientes foram mantidos em tratamento de antibioticoterapia por $3 \mathrm{a}$ 6 meses, conforme a indicação da equipe de infectologia da Instituição. Todos os pacientes foram acompanhados no período pós-operatório de forma clínica e laboratorial para avaliação de recidiva do processo infeccioso. Os pacientes que evoluíram com recidiva da infecção tiveram indicação de amputação do membro. A - Tabela 1 demonstra os dados epidemiológicos dos pacientes e caracteriza os pacientes com o tempo até o diagnóstico de infecção e o tipo de tratamento realizado.

Consideramos como tratados e em remissão da infecção os pacientes que ao final do corte do trabalho (dezembro de 
Tabela 1 Dados epidemiológicos dos pacientes e caracteriza os pacientes com o tempo até o diagnóstico de infecção e o tipo de tratamento realizado

\begin{tabular}{|c|c|c|}
\hline Sexo & $\mathbf{N}$ & $\%$ \\
\hline M & 7 & $46.7 \%$ \\
\hline $\mathrm{F}$ & 8 & $53.3 \%$ \\
\hline \multicolumn{3}{|l|}{ Idade } \\
\hline Média & 20 & \\
\hline Mínimo & 10 & \\
\hline Máximo & 31 & \\
\hline Devio-padrão & 7.2 & \\
\hline \multicolumn{3}{|l|}{ Diagnóstico } \\
\hline Osteossarcoma & 12 & $80.0 \%$ \\
\hline Sarcoma de ewing & 2 & $13.3 \%$ \\
\hline Sarcoma indiferenciado & 1 & $6.7 \%$ \\
\hline \multicolumn{3}{|l|}{ Localizacao do tumor } \\
\hline Fêmur & 12 & $80.0 \%$ \\
\hline Tíbia & 3 & $20.0 \%$ \\
\hline \multicolumn{3}{|l|}{$\begin{array}{l}\text { Tempo entre a cirurgia e o } \\
\text { diagnóstico de infecção }\end{array}$} \\
\hline$<6 \mathrm{~m}$ & 6 & $40.0 \%$ \\
\hline $6 m-12 m$ & 2 & $13.3 \%$ \\
\hline$>12 m$ & 7 & $46.7 \%$ \\
\hline \multicolumn{3}{|l|}{ Tipo de tratamento realizado } \\
\hline Somente limpeza cirúrgica & 5 & $33.3 \%$ \\
\hline $\begin{array}{l}\text { Revisão em dois tempos com } \\
\text { espaçador de cimento acrílico com } \\
\text { antibiótico }\end{array}$ & 9 & $60.0 \%$ \\
\hline Revisão em um tempo & 1 & $6.7 \%$ \\
\hline
\end{tabular}

2018) apresentavam-se clinicamente sem sinais e sintomas de processo infeccioso e controle laboratorial negativado para processo infeccioso ativo.

Os pacientes foram divididos em duas coortes retrospectivas, sendo a amputação do membro o dado de desfecho primário do estudo. Na primeira coorte alocamos os pacientes que após o tratamento (com ou sem revisão da endoprótese), evoluíram com remissão do processo infeccioso e preservação do membro. Na segunda coorte foram alocados os pacientes em que não houve êxito no controle da infecção e que foram submetidos a amputação do membro.

Foram avaliados como fatores relacionados a infecção as seguintes variáveis: sexo (masculino ou feminino), idade do paciente (até 15 anos ou acima de 15 anos), diagnóstico (osteossarcoma ou outros tumores), osso acometido (fêmur ou tíbia), duração da cirurgia de colocação da endoprótese (até $300 \mathrm{~min}$ ou acima de $300 \mathrm{~min}$ ), contagem de leucócitos no pré-operatório imediato da cirurgia de colocação da endoprótese (leucopenia até 3000 leucócitos ou não leucopenia, acima de 3000 leucócitos), infecção do trato urinário no $1^{\circ}$ ano pós-operatório da colocação de endoprótese (presença e ausência de infecção) e tempo decorrido entre a colocação da endoprótese e o diagnóstico da infecção (até $6 \mathrm{~m}$, entre $6 \mathrm{~m}$ e um ano, acima de um ano).

Foi realizado o Teste Exato de Fisher, com significância estatística de 0,05 para avaliar a significância da associação das variáveis categóricas (bicategóricas), uma vez que os grupos eram pequenos. Para a variável tricategórica foi utilizado o teste Anova. Para cada variável a hipótese nula (H0) foi de que não havia diferença entre as categorias e o desfecho de amputação.

A média de tempo entre os três grupos de pacientes, com base no status final, (revisão de endoprótese e preservação do membro, amputação do membro e limpeza cirúrgica) foi avaliada de modo separado. As médias de tempo foram comparadas pelo método de t-student.

\section{Resultados}

Dos 15 pacientes estudados, 3 evoluíram com persistência do processo infeccioso e foram submetidos a amputação do membro. A eficácia do protocolo de tratamento realizado foi de $80 \%$ para preservação do membro.

Dos fatores de risco avaliados (sexo, idade, diagnóstico, osso acometido, duração da cirurgia de colocação da prótese, contagem de leucócitos no pré-operatório imediato da cirurgia de colocação da endoprótese, infecção do trato urinário no $1^{\circ}$ ano pós-operatório da colocação da endoprótese e tempo decorrido entre a colocação da endoprótese e o diagnóstico de infecção) nenhum foi identificado estar diretamente relacionado com a amputação nos pacientes que apresentaram infecção periprótese.

A - Tabela 2 demonstra as variáveis estudadas, a incidência entre pacientes com o membro amputado e preservado, o valor do teste exato de Fisher para cada uma das variáveis e o resultado do teste Anova para o tempo entre a cirurgia de endoprótese e o diagnóstico de infecção.

Nenhum paciente que evoluiu para amputação apresentou sinais de infecção precocemente (menos de 6 meses). A média de tempo para aparecer os sinais de infecção para os pacientes que realizaram revisão e houve sucesso com a manutenção do membro foi 710 dias (158-1729 dias), para os pacientes que evoluíram para amputação foi 1246 dias (294-2352 dias) e para os pacientes que realizaram somente limpeza cirúrgica foi 199 dias (11 - 784 dias). A - Tabela 3 demonstra as médias em meses e o resultado o t-student encontrado.

Os dados demonstram haver tendência de diferença entre a média de tempo do aparecimento do processo infeccioso e o desfecho final do paciente (-Figura $\mathbf{1}$ ).

\section{Discussão}

As infecções periprótese continuam sendo uma das causas mais comuns de complicações na falência do tratamento com endoprótese. Falência, morbidade e mortalidade, são os resultados mais comuns desta afecção. Estudos recentes apontam que a infecção levará ao aumento do número de amputação após a inserção das endopróteses. ${ }^{16,17}$ 
Tabela 2 Categorias de todas as variáveis estudadas, a incidência entre pacientes com o membro amputado e preservados e o valor do teste exato de Fisher para cada uma das variáveis e o resultado do teste Anova para o tempo entre a cirurgia de endoprótese e o diagnóstico de infecção

\begin{tabular}{|c|c|c|c|}
\hline Variáveis & $\begin{array}{l}\text { Preservação } \\
\text { do Membro }\end{array}$ & Amputação & $\begin{array}{l}\text { Resultado } \\
\text { Estatístico }\end{array}$ \\
\hline Sexo & & & Fisher \\
\hline Masculino & 6 & 1 & 0.250 \\
\hline Feminino & 6 & 2 & \\
\hline Diagnóstico & & & Fisher \\
\hline Osteossarcoma & 9 & 3 & 0.875 \\
\hline $\begin{array}{l}\text { Não- } \\
\text { Osteossarcoma }\end{array}$ & 3 & 0 & \\
\hline $\begin{array}{l}\text { Osso } \\
\text { Acometido }\end{array}$ & & & Fisher \\
\hline Fêmur & 10 & 2 & 0.533 \\
\hline Tíbia & 2 & 1 & \\
\hline $\begin{array}{l}\text { Leucopenia Pre- } \\
\text { Operatoria }\end{array}$ & & & Fisher \\
\hline $\begin{array}{l}\text { acima } 3000 \\
\text { leucocitos }\end{array}$ & 6 & 2 & 0.250 \\
\hline $\begin{array}{l}\text { abaixo de } 3000 \\
\text { leucocitos }\end{array}$ & 6 & 1 & \\
\hline $\begin{array}{l}\text { ITU no } 10 \text { ano } \\
\text { Pos-Operatório }\end{array}$ & & & Fisher \\
\hline Não & 9 & 3 & 0.875 \\
\hline Sim & 3 & 0 & \\
\hline $\begin{array}{l}\text { Duracão da } \\
\text { Cirurgia }\end{array}$ & & & Fisher \\
\hline até 300 min & 4 & 2 & 1.037 \\
\hline $\begin{array}{l}\text { acima de } \\
300 \text { min }\end{array}$ & 8 & 1 & \\
\hline Idade & & & Fisher \\
\hline Até 15 anos & 5 & 0 & 1.750 \\
\hline $\begin{array}{l}\text { acima de } 15 \\
\text { anos }\end{array}$ & 7 & 3 & \\
\hline $\begin{array}{l}\text { Tempo entre } \\
\text { cirurgia e } \\
\text { diagnostico de } \\
\text { infecção }\end{array}$ & & & Anova \\
\hline$<6 \mathrm{~m}$ & 6 & 0 & 0.269 \\
\hline $6 m-12 m$ & 1 & 1 & \\
\hline$>12 m$ & 5 & 2 & \\
\hline
\end{tabular}

Alguns dos métodos de tratamento para esta complicação são: o desbrimento com a retenção e limpeza da endoprótese $(D+R)$; a revisão da endoprótese em um único estágio (na qual é realizado a retirada da prótese infectada) seguida de limpeza vigorosa e instalação de uma nova endoprótese; a revisão em dois estágios, utilizando espaçador com cimento impregnado com antibiótico e recolocação de outra
Tabela 3 Médias em meses e o resultado o t-student encontrado para cada subgrupo de pacientes

\begin{tabular}{|l|l|l|}
\hline Status & $\begin{array}{l}\text { Tempo até } \\
\text { infecção (meses) }\end{array}$ & t-student \\
\hline $\begin{array}{l}\text { Revisão com preservação } \\
\text { do Membro }\end{array}$ & 23,7 & 0,027 \\
\hline Amputação & 41,5 & 0,173 \\
\hline Somente limpeza Cirúrgica & 6,7 & 0,247 \\
\hline
\end{tabular}

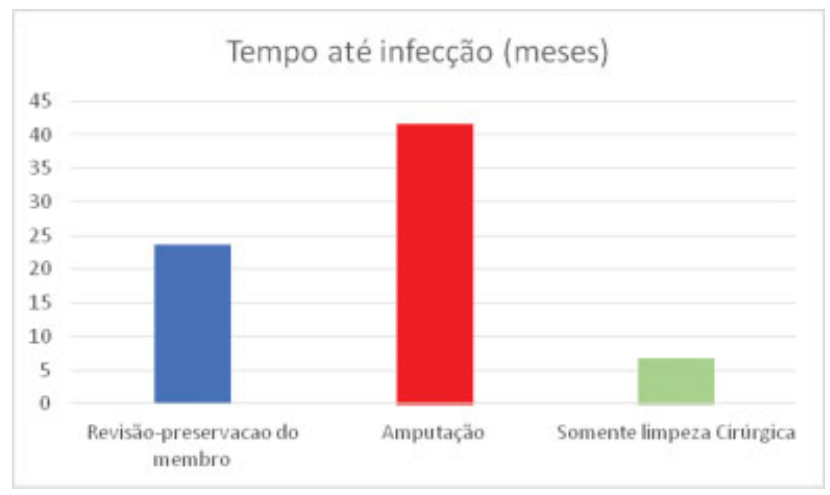

Fig. 1 Gráfico em meses do tempo até infecção para cada subgrupo de pacientes (Revisão com Preservação do Membro, Amputação, Somente limpeza Cirúrgica).

endoprótese em um segundo tempo. 0 espaçador é retirado após a normalização do quadro clínico e laboratorial do paciente, sendo então substituído pela nova endoprótese. Por fim, a amputação é um método optado na falha de todos os outros demais métodos com a finalidade de controle da doença infecciosa e preservação da vida do paciente.

Destes, a revisão em dois estágios é a que comprovadamente apresenta os melhores resultados. Nossa equipe utiliza esse método de tratamento há mais de 10 anos, com bons resultados clínicos e funcionais. ${ }^{6}$ Os trabalhos na literatura apontam de 63 a $100 \%$ de eficácia para esse método de tratamento. ${ }^{18,19}$

Na nossa casuística, os pacientes com diagnóstico de osteossarcoma foram os mais acometidos pela infecção pós operatória relacionada com a endoprótese. Estes dados corroboram com achados de Racano et al. ${ }^{5}$ que identificou o osteossarcoma como principal diagnóstico relacionado, sendo ewing e condrossarcoma os que mais se aproximam de sua estatística. ${ }^{5}$

Apesar de uma maior porcentagem dos pacientes apresentar os sintomas mais tardiamente (acima de 12 meses), os pacientes que apresentaram sintomas mais precocemente evoluíram de forma mais favorável. Esses dados confrontam com os achados de Hardes et al. ${ }^{1}$ Para os autores, a quimioterapia sozinha, assim como o tempo de em que ocorreu a infecção, virulência e o tipo de infecção não apresentam influência nos resultados finais dos pacientes. ${ }^{1}$ Já Muratori et al. $^{16}$ verificaram em seus pacientes o número alto de infecção quando estavam há mais de 12 meses em pós operatório, fato este que vai de encontro aos nossos achados. 
Apesar do fêmur ser o sítio cirúrgico de maior incidência, a localização entre fêmur e tíbia não apresenta relação com a evolução do paciente. Morii et al. ${ }^{3}$ relatam a tíbia, bem como a idade abaixo de 30 anos e o fato do tumor ser primário do osso, como achados comuns em pacientes que necessitaram revisar suas cirurgias por conta de infecção. ${ }^{3}$

Segundo Hardes et al. ${ }^{1}$ o desbridamento e a retenção do implante sem a substituição das hastes pode ser uma boa estratégia em infecções precoces $(<6 \mathrm{~m})$, entretanto, tal qual a nossa equipe, os autores tem preferência pela revisão em dois tempos para colher os melhores resultados. ${ }^{1}$

Dentre todos os tratamentos, a amputação é o modelo de tratamento visto como última arma no arsenal contra a infecção. No presente estudo, o tempo médio para a realização da amputação foi de 41,5 semanas. Antes desta técnica radical, foram tentados a limpeza com retenção e a troca do implante por espaçador, seguido pelo reimplante quantas vezes foram necessárias. Entretanto, em um grupo importante de pacientes, na falha das demais técnicas, invariavelmente, a amputação apresenta-se como última e única solução viável. ${ }^{20}$

\section{Fragilidade do estudo}

A maior fragilidade do estudo diz respeito ao baixo poder estatístico relacionado a amostra pequena. Por se tratar de evento raro, os estudos relacionados a cirurgias de endoprótese não convencional apresentam amostras reduzidas. Esse viés pode ser corrigido com estudos multicêntricos e amostras maiores. Análises maiores poderão demonstrar a importância de outros fatores de risco que não foram identificados nesse estudo.

\section{Conclusão}

Identificamos que o tempo entre a cirurgia de colocação da endoprótese e o aparecimento dos sintomas de infecção tende a ter relação com o desfecho e a evolução do paciente no tratamento da infecção peri-endoprótese. Os demais fatores associados, apesar de, aparentementemente, relacionados, não se mostraram estatisticamente relacionados à má evolução no tratamento.

\section{Suporte Financeiro}

Não houve suporte financeiro de fontes públicas, comerciais, ou sem fins lucrativos.

\section{Conflito de Interesses}

Os autores declaram não haver conflitos de interesses.

\section{Referências}

1 Hardes J, Gebert C, Schwappach A, et al. Characteristics and outcome of infections associated with tumor endoprostheses. Arch Orthop Trauma Surg 2006;126(05):289-296
2 Torbert JT, Fox EJ, Hosalkar HS, Ogilvie CM, Lackman RD. Endoprosthetic reconstructions: results of long-term followup of 139 patients. Clin Orthop Relat Res 2005;438(438):51-59

3 Morii T, Morioka H, Ueda T, et al. Deep infection in tumor endoprosthesis around the knee: a multi-institutional study by the Japanese musculoskeletal oncology group. BMC Musculoskelet Disord 2013;14:51

4 Peel T, May D, Buising K, Thursky K, Slavin M, Choong P. Infective complications following tumour endoprosthesis surgery for bone and soft tissue tumours. Eur J Surg Oncol 2014;40(09): 1087-1094

5 Racano A, Pazionis T, Farrokhyar F, Deheshi B, Ghert M. High infection rate outcomes in long-bone tumor surgery with endoprosthetic reconstruction in adults: a systematic review. Clin Orthop Relat Res 2013;471(06):2017-2027

6 Viola DCM, Cardozo Filho NS, Nunes RT, et al. O uso de espaçadores com antibiótico no tratamento das infecções em endopróteses de joelho. Acta Ortop Bras 2009;17(03):144-148

7 Zimmerli W, Trampuz A, Ochsner PE. Prosthetic-joint infections. N Engl J Med 2004;351(16):1645-1654

8 Deva AK, Adams WP Jr, Vickery K. The role of bacterial biofilms in device-associated infection. Plast Reconstr Surg 2013;132(05): 1319-1328

9 Dhanoa A, Ajit Singh V, Elbahri H. Deep Infections after Endoprosthetic Replacement Operations in Orthopedic Oncology Patients. Surg Infect (Larchmt) 2015;16(03):323-332

10 Allison D, Huang E, Ahlmann ER, Carney S, Wang L, Menendez LR. Peri-Prosthetic Infection in the Orthopedic Tumor Patient. JISRF Reconstr Rev 2014;4(03):13-22

11 Nagaya LH, Salles MJC, Takikawa LSC, et al. Infections after shoulder arthroplasty are correlated with higher anesthetic risk score: a case-control study in Brazil. Braz J Infect Dis 2017;21(06): 613-619

12 Wafa H, Grimer RJ, Reddy K, et al. Retrospective evaluation of the incidence of early periprosthetic infection with silver-treated endoprostheses in high-risk patients: case-control study. Bone Joint J 2015;97-B(02):252-257

13 Tande AJ, Patel R. Prosthetic joint infection. Clin Microbiol Rev 2014;27(02):302-345

14 Zajonz D, Zieme A, Prietzel T, et al. Periprosthetic joint infections in modular endoprostheses of the lower extremities: a retrospective observational study in 101 patients. Patient Saf Surg 2016;10:6

15 Sigmund IK, Gamper J, Weber C, et al. Efficacy of different revision procedures for infected megaprostheses in musculoskeletal tumour surgery of the lower limb. PLoS One 2018;13(07):e0200304

16 Muratori F, Mondanelli N, Prifti X, et al. Total femur prosthesis in oncological and not oncological series. Survival and failures. J Orthop 2019;17:215-220

17 Prabowo Y, Steven P. Management of infected megaprosthesis with debridement and implant preservation using glutaraldehyde. J Indon Orthop Traumatol 2018;1(01):33-39

18 Zuidhof RJ, Lowik C, Ploegmakers J, Wouthuyzen-Barkker M, Jutte P. Periprosthetic joint infection in orthopaedic surgical oncology. Ann Joint 2019;4:26-26

19 Niccoli G, Mercurio D, Cortese F. Bone scan in painful knee arthroplasty: obsolete or actual examination? Acta Biomed 2017;88(2S):68-77

20 Pilge H, Gradl G, von Eisenhart-Rothe R, Gollwitzer H. Incidence and outcome after infection of megaprostheses. Hip Int 2012;22 (Suppl 8):S83-S90 